DOl: $10.30972 /$ eitt.704769

\title{
Flujo viscoso: diseño y construcción de un dispositivo para determinar el coeficiente de viscosidad dinámica del agua
}

\author{
Lucero Delugo*, Ing. Cesar A. Cabrera* y Dr. Patricio F. Provasi*
}

\section{Resumen}

La viscosidad dinámica es una propiedad de los fluidos que caracteriza la resistencia a fluir, es decir el equivalente al rozamiento entre sólidos y se debe principalmente a las fuerzas de adhesión y cohesión existente entre las moléculas que los componen y con las del contenedor. El conocimiento del valor de esta propiedad para cada fluido es de importancia en diversos procesos industriales, físicos y, por supuesto, biológicos. Considerando además la escasez de experimentos para determinar la viscosidad de líquidos con coeficientes bajos, en este trabajo se diseñó y construyó un dispositivo para el estudio y determinación de la viscosidad del agua con la finalidad de que sea utilizado en propuestas de trabajo experimental para alumnos del nivel secundario.

Palabras claves: Flujo viscoso, ley de Poiseuille, viscosidad del agua.

\section{Abstract}

The dynamic viscosity is a property of fluids that characterizes resistance to flow, that is, the equivalent of friction between solids and is mainly due to the adhesion and cohesion forces existing between the molecules that compose them and 
with those of the container. Knowledge of the value of this property for each fluid is of importance in various industrial, physical and, of course, biological processes. Considering also the scarcity of experiments to determine the viscosity of liquids with low coefficients, in the presented work a device was designed and built for the study and determination of the viscosity of water in order to be used in experimental work proposals for high school students.

Keywords: Poiseuille’s law, viscous flow, water's viscosity.

\section{Introducción}

El término viscosidad se utiliza para describir la resistencia al movimiento relativo que presentan dos capas adyacentes del fluido o bien entre el líquido y algún sólido. Esta propiedad de los fluidos es causada por las fuerzas de cohesión y adhesión intermoleculares o en esencia las fuerzas electromagnéticas intermoleculares.

La resistencia a fluir es de suma importancia ya que debido a ella parte de la energía cinética de un fluido se convierte en energía térmica. Pero también, resulta impensable la existencia de organismos vivos en un espacio sin resistencias al movimiento.

Así, dos de los ejemplos más frecuentemente citados son el sistema circulatorio en animales [1] y en plantas [2] donde se observan implementaciones muy ingeniosas de reproducción de algunas de las características reales de estos procesos tan complejos, aunque comunes para la naturaleza. Otros ejemplos se pueden ver en la Referencia [3].

Existen diversos tipos de dispositivos para determinar la viscosidad de un líquido. Entre ellos se observan los que consideran un cuerpo sólido moviéndose en el fluido, es decir, viscosímetros de cuerpo móvil, cuyos abanderados son los de caída libre de esferas que funcionan según la ley de Stokes y son prácticos para viscosidades altas. También se pueden ver viscosímetros rotacionales [4], por escurrimiento [5], sifones [6], péndulos [7 ], los de circulación capilar [8], que son más prácticos para viscosidades bajas. 


\section{Aspecto Teórico}

Es importante remarcar que la diferencia entre coeficiente de viscosidad dinámica, $\mathbf{\eta}[\mathrm{mPa} \cdot \mathrm{s}]$, y cinemático, $\mathbf{v}=\mathbf{\eta} / \boldsymbol{\rho}\left[\mathrm{mm}^{2} \cdot \mathrm{s}^{-1}\right]$, radica fundamentalmente en que el primero nos da información sobre la fuerza necesaria para hacer fluir el fluido y el segundo nos informa qué tan rápido se mueve éste cuando se aplica una fuerza.

Si se considera un tubo cilíndrico de radio $\mathbf{r}$ y longitud $\mathbf{L}$, a través del cual circula un líquido newtoniano en régimen laminar, estacionario y con un caudal $\mathbf{Q}$, debido a una diferencia de presión $\Delta \mathbf{P}$, y siendo que el líquido posee una viscosidad dinámica uniforme caracterizada por $\mathbf{\eta}$. La expresión para el movimiento del líquido se puede escribir:

$$
Q=\frac{\Delta P \pi r^{4}}{8 \eta L}
$$

Que recibe el nombre de ley de Pouiseuille. Esta expresión se puede abreviar como:

$$
R Q=\Delta P
$$

La analogía con la ley de Ohm, o la transmisión del calor, entre otras tantas, resulta evidente. Claro, esta igualdad de forma está, en principio, restringida a la aproximación lineal de las mismas. Pero resulta de interés para ejercitar la memoria [9] (el movimiento del flujo de la sustancia de interés multiplicado por la resistencia al mismo es igual a la fuerza impulsora) y proponerle al estudiante, neófito, ejercicios de análisis.

La similitud en formas de algunas de las leyes de la naturaleza puede aplicarse en otros campos como ser, por ejemplo, el análogo hidráulico al equilibrio secular en física nuclear [10].

Uno de estos ejercicios de análisis es el que se desarrolla en el presente trabajo, de forma que si se hace circular un fluido por un tubo de dimensiones conocidas y se mide tanto el caudal como la caída de presión, se puede determinar la resistencia y por ende el coeficiente de viscosidad dinámica del fluido. Además, si este líquido fluye a través del tubo sólo por acción de la gravedad, la ecuación (1) se puede expresar como 


$$
Q=\frac{\rho g \Delta h \pi r^{4}}{8 \eta L}
$$

siendo que $\boldsymbol{\rho}$ es la densidad del fluido, $\mathbf{g}$ es la aceleración de la gravedad y $\Delta \mathbf{h}$ la diferencia entre las alturas de las columnas de líquido.

Vale la pena aclarar en este punto que las consideraciones hechas al comienzo del ítem no son menores y que en el caso de no cumplirse, aunque sea levemente, los resultados pueden cambiar drásticamente.

El coeficiente de viscosidad dinámica del agua no depende tan fuertemente de la temperatura en un régimen turbulento como lo hace en el régimen laminar. Lo mismo ocurre si el capilar utilizado fuese muy delgado entonces estaríamos afuera de la aproximación de líquido newtoniano, o si hubiera concentraciones altas de algún soluto disuelto en el agua harían cambiar el valor del coeficiente.

\section{Diseño Experimental}

\section{- Dispositivo Experimental}

El dispositivo fue diseñado específicamente para líquidos con bajo coeficiente de dilatación y tomando como modelos el viscosímetro de tubo capilar [7]. Es valioso admitir que el modelo propuesto en [7(b)] es el más sencillo para hacer y utilizar como demostración en un aula.

Los materiales utilizados para la construcción del mismo fueron: dos tubos de policloruro de vinilo (PVC), cada uno con sus respectivas tapas en sus extremos inferiores, una manguera de nivelación y un recipiente de medidas graduado.

Los tubos poseen longitudes de 22,6 \pm 0,1 cm y 34,3 \pm 0,1 cm y diámetros iguales a $100 \mathrm{~mm}$. El diámetro y longitud de la manguera son respectivamente de 0,6 \pm 0,1 $\mathrm{cm}$ y 89,7 \pm 0,3 $\mathrm{cm}$. El recipiente graduado tiene una capacidad de $500 \mathrm{~cm} 3$.

El esquema del dispositivo se muestra en la Figura 1. Ambos tubos fueron perforados a una altura de 8 centímetros desde su base. El diámetro de las perforaciones 
fue lo suficiente para que permitiera insertar la manguera a través de estas. Luego, para evitar pérdidas, se aplicó una capa de pegamento especial para tubos de PVC en la sección de unión entre la manguera y el tubo.

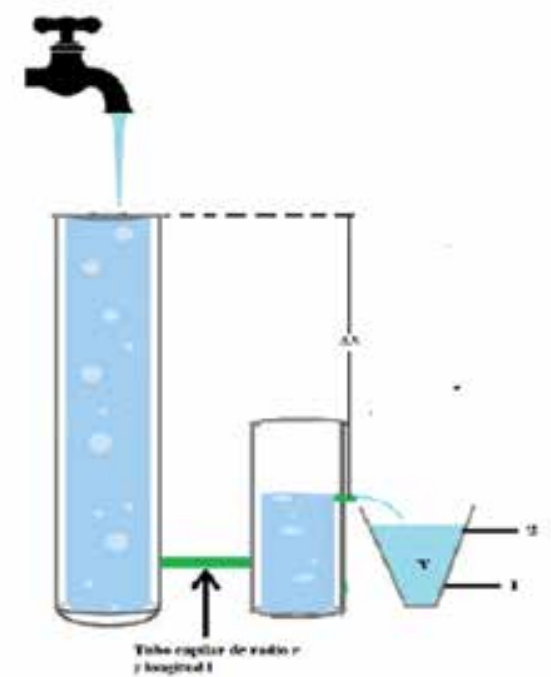

Figura 1: Esquema del dispositivo en funcionamiento. El agua circula de izquierda a derecha. La manguera verde que une los dos tubos es en la que se mide la resistencia o el coeficiente de viscosidad.

En el tubo más corto se realizó una segunda perforación a una altura mayor, cerca del borde superior, en la que se insertó un pequeño recorte de manguera de nivelación con la finalidad de conducir el agua hacia el recipiente graduado.

La longitud de la manguera que une los tubos fue medida incluyendo los segmentos que sobresalen desde las preformaciones hacia el interior de los tubos.

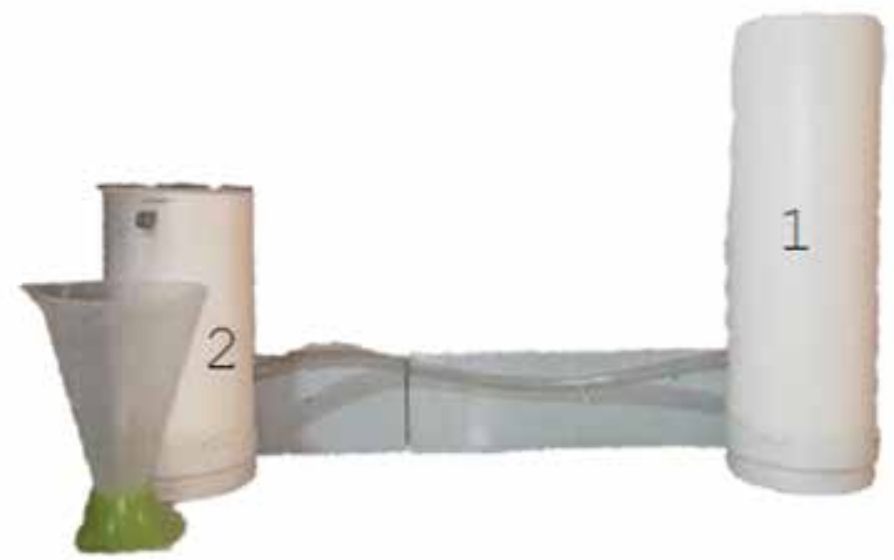

Figura 2: Fotografía del dispositivo con el recipiente graduado en frente del tubo 2. El agua circula de derecha a izquierda. La manguera transparente que une ambos tubos es en la que se mide la viscosidad del agua 
La diferencia de presión es directamente proporcional a la diferencia de alturas entre las columnas de ambos tubos y se mantiene constante, manteniendo abierta la canilla y, para el caso de un caudal muy grande proveniente de la red se practica un desagote en el tubo más alto o bien se deja que rebalse.

\section{- - Realización de la experiencia.}

El viscosímetro se situó bajo un grifo conectado a la red domiciliaria de agua. La salida de agua desde el grifo se mantuvo tal que posea un flujo constante y que las alturas de los niveles de agua en ambos tubos permanezcan estables, es decir, en un régimen estacionario.

La altura del nivel de agua del tubo más alto y que se encontraba bajo el grifo se estableció en 25,0 \pm 0,1 cm. El nivel de agua del tubo más bajo se estableció a una altura de 12,0 $\pm 0,1 \mathrm{~cm}$, que es igual a la altura a la que sale el agua del sistema y fluye hacia el recipiente graduado. Las alturas fueron medidas desde los orificios en donde se insertaron los extremos de la manguera de nivelación hasta el nivel de agua correspondiente.

Una vez constatado el régimen estacionario se procede a hacer la medición del caudal, cronometrando el volumen de agua con el recipiente graduado de $500 \mathrm{~cm} 3$. Esto se logró con un cronómetro puesto al lado del recipiente graduado y filmando el proceso, para evitar tiempos de retardo asociados a los reflejos del operador.

Para los cálculos del error se consideró el valor de densidad del agua tabulado de acuerdo a la temperatura de la misma al momento de la medición y el valor de la aceleración de la gravedad correspondiente a la Ciudad de Corrientes.

\section{Resultados y Discusión}

A partir de los datos registrados de las mediciones, se determinó un valor de 0,001 $\pm 0,0003[\mathrm{~Pa} \cdot \mathrm{s}]$ para el coeficiente de viscosidad dinámica del agua. El resultado obtenido tiene un error de 7,6 \% respecto al valor de tabla para la viscosidad del agua a la temperatura de $24^{\circ} \mathrm{C}$ que es de $0.00092933[\mathrm{~Pa} \cdot \mathrm{s}] \mathrm{o}\left[\mathrm{kg} \mathrm{m}^{-1} \mathrm{~s}^{-1}\right]$. 
Hay varios factores que pueden introducir error en este experimento, uno de ellos es el valor de densidad del agua que se considera y el cual debería ser el que corresponde a la temperatura del experimento. En nuestro caso la temperatura fue de $24,3^{\circ} \mathrm{C}$ lo que establece la densidad del agua en $0,9974 \mathrm{~g} / \mathrm{cm}^{3}$. Por otro lado, el flujo no es perfectamente laminar y nuestra estimación del número de Reynolds, $\mathbf{N R}=\mathbf{2} \cdot \mathbf{r} \cdot \boldsymbol{\rho} \cdot \mathbf{v} / \mathbf{\eta}$, nos da 2550 cuando usamos el coeficiente que obtuvimos experimentalmente y 2758 cuando usamos el valor de tabla. Es decir, tenemos un flujo apenas turbulento. Lo que puede explicar la desviación en el valor obtenido experimentalmente para el coeficiente de viscosidad.

\section{Conclusiones}

El presente trabajo consistió en el diseño y construcción de un dispositivo capaz de utilizarse en la determinación del coeficiente dinámico de viscosidad de líquidos con coeficientes bajos, en particular, el agua.

Si bien este dispositivo es algo más elaborado que el de la Ref. [8(b)] que es adecuado para una demostración práctica y rápida en un salón de clases, nos permite hacer estimaciones relativamente precisas del coeficiente de viscosidad dinámica, que lo vuelve ideal para las clases en el laboratorio. Además su fácil armado y el bajo coste de los materiales utilizados permiten la construcción de varios viscosímetros cuyos componentes difieran en sus dimensiones para así proponer a distintos grupos de alumnos trabajar con ellos y comparar los resultados obtenidos como también señalar y explicar las diferencias observadas en los caudales de los fluidos que se encuentra en uno y otro dispositivo.

También, como puede verse, nos permite realizar la medición del número de Reynolds, con muy buena aproximación. 7,5\%. Inclusive nos permitirá visualizar el comportamiento del flujo caracterizado por él al insertar con una jeringa algún colorante en la boca de entrada de la manguera en la cual medimos la resistencia hidráulica o el coeficiente de viscosidad.

Un factor importante de remarcar es la analogía que se logra con este dispositivo entre leyes de igual forma, en particular la analogía con la ley de Ohm es evidente. 
Esto nos lleva a pensar en probar con conexiones en serie y paralelo para las mangueras, las que serían de fácil implementación con conexiones tipo T.

\section{Referencias}

[1] (a) J. Steketee "Demonstration model of the human circulation" Eur. J. Phys. 2 (1981) 150-154. (b) Benjamin Spitznagel, Justin Weigal, and Juan Rodriguez "Visualizing Viscous Flow and Diffusion in the Circulatory System" Phys. Teach. 57 (2019) 529-532.

[2] M. Denny "Tree hydraulics: how sap rises" Eur. J. Phys. 33 (2012) 43-53.

[3] D. C. Agrawaly and V. J. Menonz "Thanks to the three viscous formulae" Phys. Educ. 34 (1999) 149-152.

[4] Y. Kraftmakher "Rotational viscometers - a subject for student projects" Phys. Educ. 45 (2010) 622-628.

[5] (a) A. T. Widdicombe, P. Ravindrarajah, A. Sapelkin, A. E. Phillips, D. Dunstan, M. T. Dove, V. V. Brazhkin and K. Trachenko "Measurement of bitumen viscosity in a room-temperature drop experiment: student education, public outreach and modern science in one" Phys. Educ. 49 (2014) 406-411. (b) I. A. Sianoudis and E. Drakaki “An approach to Poiseuille's law in an undergraduate laboratory experiment” Eur. J. Phys. 29 (2008) 489-495.

[6] (a) H. S. Badeer and C. E. Synolakis "The Bernoulli-Poiseuille equation" Phys. Teach. 27 (1989) 598-601. (b) H. S. Badeer and J. W. Hicks "Role of viscous resistance in siphon flow" Phys. Teach. 28, 558 (1990) 558-559.

[7] J. Costa Leme, and A. Oliveira "Pendulum Underwater - An Approach for Quantifying Viscosity” Phys. Teach. 55 (2017) 555-557.

[8] (a) T. Massalha and R. M Digilov "Capillary viscometer with a pressure sensor: a subject for student projects” Eur. J. Phys. 36 (2015) 065045 (9pp). (b) R. D. Edge "Viscosity" Phys. Teach. 20 (1982) 47-48. (c) F. M. Ortega, O. D. Pavioni and H. L. Domínguez “A Communicating-Vessel Viscosimeter” Phys. Teach. 45 (2007) 116-118. (d) S. Chakrabarti "A novel experimental setup to study the Hagen-Poiseuille and Bernoulli equations for a gas and determination of the viscosity of air" Eur. J. Phys. 36 (2015) 065046 (14pp).

[9] D. E. Rehfuss “Current Concepts Consolidated” Phys. Teach. 42 (2004) 103-107.

[10] Thomas B. Greenslade "Simulated secular equilibrium" Phys. Teach. 40 (2002) 21-23. 